\title{
The Role of Chronic Inflammation in Prostate Carcinogenesis: A Follow-Up Study
}

\author{
Wei Chen $^{1^{*}}$, Liwei Jia ${ }^{1^{*}}$, Sanjay Gupta ${ }^{2,3,4}$, Gregory T. MacLennan ${ }^{1,2,3}$
}

Cite this article: Chen W, Jia LW, Gupta S, MacLennan GT: The role of chronic inflammation in prostate carcinogenesis: A follow-up study. Ann Urol Oncol 2019; 2(1): 1-8. https://doi.org/10.32948/ auo.2019.01.14

\begin{abstract}
Purpose To investigate the role of chronic inflammation in prostatic carcinogenesis with an emphasis on cancers of grade group 2 or above.

Methods The presence/absence and extent of chronic inflammation and other relevant pathological findings were assessed using prostate needle biopsies obtained from patients with clinical parameters with suspicion of malignancy. In patients with no prior prostate cancer, follow-up biopsies were reviewed and correlated with the initial pathological findings.

Results Of 1,006 prostate needle biopsy cases accessioned over 3 years at our institution, the initial biopsies of 244 cases were identified with no evidence of prostate cancer. These cases were divided into two subsets, including 202 cases with and 42 without chronic inflammation. Pathological findings assessed in this subset included post-atrophic hyperplasia, proliferative inflammatory atrophy, and high-grade prostatic intraepithelial neoplasia (HGPIN). Postatrophic hyperplasia and proliferative inflammatory atrophy were noted only in patients with chronic inflammation. HGPIN was identified in 22 of the initial biopsy cases, specifically in $21(10.3 \%)$ cases with inflammation and one case $(2.4 \%)$ without inflammation. In followup biopsies, 70 patients $(34.7 \%)$ with chronic inflammation were found to have prostatic adenocarcinoma with Gleason pattern $3+3(42.9 \%$; grade group 1), Gleason pattern $3+4$ (24.3\%; grade group 2), Gleason pattern $4+3(10 \%$; grade group 3) and Gleason scores of 8 or higher (22.8\%; grade groups 4 or 5$)$, whereas cancer was found in 10 patients $(23.8 \%)$ without chronic inflammation in the initial biopsy. Patients whose initial biopsies were benign and without inflammation did not show evidence of high-grade cancer (Gleason score of 8 or higher). Closely encroaching inflammation was observed more frequently in cancers of grade group 2 or above $(76.5 \%, 13 / 17)$ compared with grade group $1(50.0 \%, 3 / 6)$.

Conclusions Our findings provide additional data supporting a role for chronic inflammation in the development of prostatic adenocarcinoma.
\end{abstract}

Key words chronic inflammation, prostate cancer, needle biopsy

1. Department of Pathology, Case Western Reserve University \& University Hospitals Cleveland Medical Center, Cleveland, Ohio, USA.

2. Department of Urology, Case Western Reserve University \& University Hospitals Cleveland Medical Center, Cleveland, Ohio, USA.

3. Division of General Medical Sciences, Case Comprehensive Cancer Center, Cleveland, Ohio, USA.

4. Department of Urology, Louis Stokes Cleveland Veterans Affairs Medical Center, Cleveland, Ohio, USA.

*: W. Chen and LW. Jia contributed equally to this work.

Correspondence: Gregory T. MacLennan (Pathology Residency Training Program Case Western Reserve University \& University Hospitals Cleveland Medical Center, 11100 Euclid Ave. Cleveland, OH 44106; Email: Gregory.MacLennan@UHHospitals.org). 


\section{Introduction}

Prostatic adenocarcinoma is the most common visceral cancer in males in the United States [1]. Although considerable progress has been achieved in understanding the progression of prostate cancer, the key drivers of its initiation and development, etiology and risk factors are not fully understood.

Persistent chronic inflammation has emerged as a potential risk factor in the development of carcinoma of several organs, including the liver, colon, urinary bladder and pancreas [2-4]. It has been shown to contribute to malignant biological behavior during disease initiation, growth in a localized environment, tumor cell invasion, angiogenesis, and metastasis [5]. The high prevalence of chronic inflammation in prostate needle biopsies and surgically excised specimens has prompted studies of a possible association between chronic inflammation and prostatic adenocarcinoma [6]. Prostatic inflammation may stem from many possible etiologies, including bacterial or viral infections, dietary factors, hormonal influences, autoimmune responses and urine reflux into prostatic ducts [5]. It has been postulated that acinar or ductal epithelium continuously exposed to the deleterious effects of chronic inflammation undergoes progressive genetic changes that result in high-grade prostatic intraepithelial neoplasia (HGPIN), which is the putative precursor to prostate cancer [7]. Whether the presence or extent of chronic inflammation in benign prostate tissue influences the development of prostatic adenocarcinoma is controversial. There is little information regarding whether the grade and/or biologic aggressiveness of prostate cancers is influenced by concomitant inflammation. We designed a follow-up study separating patients into two groups based on the inflammation status of the initial prostate biopsies performed to diagnose prostate cancer. The association between the presence or absence of chronic prostatic inflammation in initially benign prostate needle biopsies and the subsequent development of prostatic adenocarcinoma in biopsies obtained at least one year later was investigated. The Gleason patterns and scores of cancers arising in the two different groups were compared. We also analyzed the distance between visible stromal inflammation and malignant foci.

\section{Materials and methods}

\section{Patient selection}

Needle biopsies were performed at the University Hospitals Cleveland Medical Center (Cleveland, OH, USA). Specimens from all ethnic groups were included. This study was approved by the Institutional Review Board of University Hospitals (IRB number 02-14-36). A total of 1,006 prostate biopsy cases accessioned in the Department of Pathology at the University Hospitals between 01/01/2007 and 12/31/2009 were studied. Of these, patients with initially benign prostate biopsies followed by at least one set of prostate biopsies performed one year or more after the initial biopsies were included in the study. Patients were excluded if the initial biopsies showed prostatic adenocarcinoma, atypical small acinar proliferation (ASAP), neuroendocrine carcinoma, intraductal adenocarcinoma or urothelial carcinoma. Patients whose subsequent biopsies were performed less than one year after the initial biopsies or who had no subsequent biopsies at our institution were also excluded. Analyses included age, ethnicity, diagnosis and years of follow-up after the initial biopsies. The study design flow chart is summarized in Figure 1.

\section{Initial and follow-up biopsies}

Transrectal prostate biopsies were performed to evaluate patients

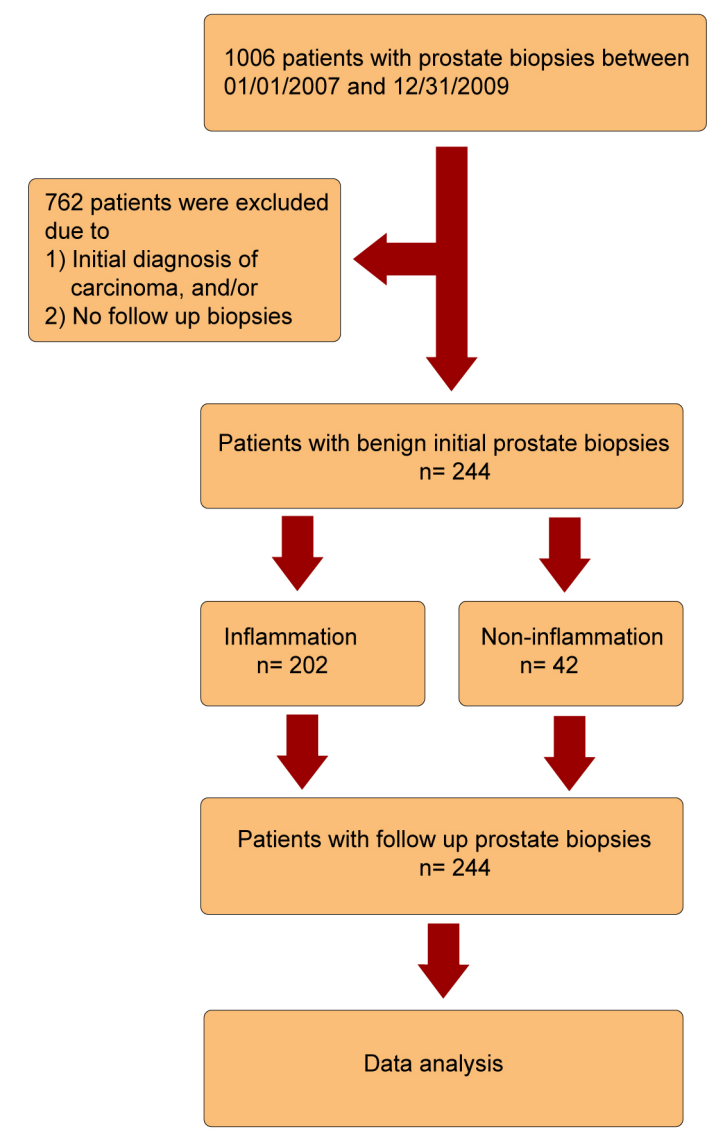

Figure 1. Flow chart of the study design.

with significantly elevated serum PSA levels and/or abnormal findings upon digital rectal examination. In all cases, at least 12 cores of prostate tissue were submitted by the surgeon, and in some cases, the number exceeded 12. Tissue specimens were fixed in $10 \%$ buffered formalin, processed, and subjected to hematoxylin and eosin (H\&E) staining according to the routine protocol. All case slides were reviewed by two or more experienced pathologists, and a consensus was achieved regarding the criteria for the various pathological diagnoses assigned to these cases. Findings were entered in a common database. Initial and follow-up biopsies were evaluated for the presence or absence of malignancy, the presence and extent of chronic inflammation, and any other significant morphological findings. In follow-up biopsies showing adenocarcinoma, the spatial relationships between adenocarcinoma and stromal inflammation were analyzed.

\section{Evaluation of chronic inflammation}

In the initial biopsy set, we defined the presence of chronic inflammation as the involvement of $1 \%$ or more of the surface area of the prostatic tissue by a chronic inflammatory cell infiltrate. The degree of inflammation was assigned according to the approximate percent of stroma and glands in the biopsy specimen involving aggregates of 50 or more lymphocytes, or by granulomatous inflammation, using the following scale: mild inflammation - $1 \%$ to $5 \%$; moderate inflammation $-5 \%$ to $50 \%$; severe inflammation greater than $50 \%$ involvement.

\section{Morphological findings in initial biopsies}

For evaluations of malignancy and chronic inflammation, all biopsies were assessed for the presence or absence of post-atrophic 
Table 1. Demographic information of patients with and without initial chronic inflammation

\begin{tabular}{llll}
\hline Items & With inflammation & Without inflammation & P value \\
\hline Case(n) & 202 & 42 & 0.003 \\
Age (year, mean) & 64.5 & 60.7 & 0.638 \\
White(n, \%) & $91(45.0)$ & $22(52.4)$ & - \\
Caucasian(n, \%) & $48(23.8)$ & $9(21.4)$ & - \\
African American(n, \%) & $57(28.2)$ & $9(21.4)$ & - \\
Asian(n, \%) & $2(1.0)$ & $0(0)$ & 0.00 \\
Other(n, \%) & $4(2.0)$ & $2(4.8)$ & $<0.05$ \\
With PIA/PAH (n, \%) & $58(28.0)$ & $0(0)$ & 0.308 \\
With PIN (n, \%) & $21(10.4)$ & $1(2.4)$ & $5.52(1-14)$ \\
Follow up years(mean, range) & $4.97(1-16)$ & . & \\
\hline
\end{tabular}

PIA, proliferative inflammatory atrophy; PAH, post-atrophic hyperplasia; PIN, prostatic intraepithelial neoplasia.

hyperplasia (PAH), proliferative inflammatory atrophy (PIA) and high-grade prostatic intraepithelial neoplasia (HGPIN). The morphological characteristics of these entities are well described in standard pathology reference texts $[8,9]$.

\section{Follow-up biopsies and adenocarcinoma diagnoses}

In follow-up biopsies, all specimens were examined for prostatic adenocarcinoma. For each adenocarcinoma case, the Gleason patterns and scores were evaluated and recorded. In compliance with the currently accepted the international society of urological pathology (ISUP) grading system for prostate cancer, cases fell into several subsets: grade group $1(3+3=6)$, grade group $2(3+$ $4=7)$, grade group $3(4+3=7)$, and a combined subset including grade groups 4 or $5(4+4=8$ or higher) [10].

Spatial relationships of adenocarcinoma and inflammation

The spatial relationships between areas of adenocarcinoma and foci of inflammation, when present in the same biopsy core, were assessed and recorded. Cases with these findings were subclassified into 3 groups according to the microscopic distance between the tumor and inflammation: 1) adjacent (inflammatory cells mixed with tumor cells); 2) tumor and inflammation within 1 $\mathrm{mm}$ of each other; 3) tumor and inflammation separated by more than $1 \mathrm{~mm}$.

\section{Statistical Analysis}

Statistical analyses were performed with SPSS 16.0 (SPSS Inc., Chicago, IL). Differences between initial and follow-up values were analyzed by the McNamara chi-square test. Bilateral logistic regression analyses were performed to evaluate the influence of chronic inflammation, age, ethnic groups and follow-up years on the development of prostatic adenocarcinoma. A $p<0.05$ was considered statistically significant.

\section{Results}

\section{Demographic information}

Of 1006 patients who underwent prostate needle biopsies between 01/01/2007 and 12/31/2009 at the University Hospitals Cleveland Medical Center, no malignancy was identified in the

Table 2. Adenocarcinoma with Gleason patterns in patients with and without initial inflammation

\begin{tabular}{llll}
\hline Items & Types of $\operatorname{cases}(\mathbf{n})$ & With inflammation(n, \%) & Without inflammation(n, \%) \\
\hline Total case & - & 202 & 42 \\
& None (164) & $133(65.3)$ & $32(76.2)$ \\
Adenocarcinoma & Present (80) & $70(34.7)$ & $10(23.8)$ \\
& $3+3(37)$ & $30(14.9)$ & $7(16.7)$ \\
Gleason Patterns & $3+4(19)$ & $17(8.4)$ & $2(4.8)$ \\
& $4+3(8)$ & $7(3.5)$ & $1(2.4)$ \\
& $4+4$ or above $(16)$ & $16(7.9)$ & $0(0.0)$ \\
\hline
\end{tabular}


Table 3. Relative risk (RR) of high grade adenocarcinoma (Gleason score over 8) in 244 patients with and without baseline inflammation

\begin{tabular}{lllll}
\hline Items & Cases(n) & No cancer or grade group 1 (n, \%)* & Grade group 2 or above (n, \%)* & RR(95\% CI)** \\
\hline Total Cases & - & 201 & 43 & - \\
Without Inflammation & 42 & $39(92.9)$ & $3(7.1)$ & 1.0 \\
With Inflammation & 202 & $162(80.2)$ & $40(19.8)$ & $3.23(0.95-10.86)$ \\
\hline
\end{tabular}

*: Adenocarcinoma in subsequent biopsies; **: Compared with patients without initial inflammation. RR, relative risk; CI, confidence interval.

initial biopsies of 244 patients. These 244 cases were divided in two subsets composed of 202 patients with inflammation and 42 patients without chronic inflammation in their initial biopsies (Table 1). The inflammation group had an older mean age than the non-inflammation group (64.5 vs $60.7, \mathrm{p}=0.003)$. The ethnic distribution between the two groups was similar. Whites and Caucasians accounted for nearly three quarters of all the patients in both subsets, African American men accounted for approximately one quarter, and Asian and other ethnic groups accounted for less than $5 \%$. No statistically significant difference was found in terms of the ethnic distribution $(p=0.64)$. All patients in the study were followed up for at least one year, with mean follow-up durations of 4.97 and 5.52 years for the inflammation and non-inflammation group, respectively $(\mathrm{p}<0.05)$.

\section{Histologic findings on initial biopsies}

In addition to chronic inflammation, the presence or absence of PAH, PIA and HGPIN was recorded in all initial biopsies. PAH and/or PIA were noted exclusively in cases with chronic inflammation $(28.8 \%$ vs $0 \%, \mathrm{p}=0.00)$. HGPIN was identified in 22 of the initial biopsy cases, specifically in 21 cases with inflammation and in one case without inflammation $(10.4 \%$ vs $2.4 \%, \mathrm{p}<0.05$ ). (Table 1 ).

Frequency of malignancy development in patients with and without initial inflammation

Follow-up biopsies were performed in 244 patients at least one year or more after their initial benign biopsies. Of the 202 patients with chronic inflammation in their initial benign biopsies, 70 patients $(34.7 \%)$ were diagnosed with adenocarcinoma in subsequent biopsies, and cancer was found in 10 patients $(23.8 \%)$ in the group of 42 men without chronic inflammation in their initial benign biopsies (Table 2). Compared to the non-inflammation group, patients with chronic inflammation in the initial biopsy had a 1.65-times higher risk of developing adenocarcinoma during follow-up [95\% confidence interval (CI) 0.74-3.67]. After adjustment for age, ethnic group, PIN status, and follow-up years, the relative risk was 1.32 (95\% CI 0.53-3.42).

We further analyzed the Gleason patterns and scores of the cancers that developed in each group. Of the cancers that arose in 70 patients who initially had chronic inflammation, 30 (42.9\%) were grade group 1, 17 (24.3\%) were grade group 2, 7 (10.0\%) were grade group 3, and $16(22.9 \%)$ were grade groups 4 or 5 . Of the cancers that arose in 10 patients who did not initially have chronic inflammation, 7 (70.0\%) were in grade group 1, 2 (20.0\%) were in grade group 2, $1(10.0 \%)$ was in grade group 3, and none were in grade groups 4 or 5 . Notably, none of the patient with initial biopsies that were free of chronic inflammation developed cancer with a Gleason score of 8 or higher. Compared with patients without inflammation in their initial biopsies, patients with initial inflammation had a 3 times increased risk of developing cancers of grade group 2 or higher [RR 3.23, 95\% CI (0.95-10.86)]. After adjusting for age, ethnic group, PIN status and follow-up years, the RR was 2.43 (95\% CI 0.58-9.33) (Table 3).

\section{Extent of chronic inflammation with adenocarcinoma}

We studied the relationship between the extent of chronic inflammation in initial biopsies and the cancers that subsequently arose in 80 patients (70 in the inflammation group and 10 in the non-inflammation group) (Table 4). The majority $(\mathrm{n}=$ 62) exhibited mild inflammation in initial biopsies (77.5\%), 7 $(8.8 \%)$ had moderate inflammation (5-50\%), 1(1.2\%) had severe inflammation, and $10(12.5 \%)$ had no inflammation in their initial biopsies. Compared with patients without inflammation in their initial biopsies, patients with mild chronic inflammation had a higher risk of developing prostate cancer during follow-up (RR $1.89,95 \%$ CI $0.87-4.11)$, particularly adenocarcinoma of grade

Table 4. Relative risk (RR) of adenocarcinoma according to inflammation status and extent in baseline prostatic biopsy

\begin{tabular}{lllll}
$\begin{array}{l}\text { Classification of } \\
\text { inflammation }\end{array}$ & Adenocarcinoma(n) & RR(95\% CI)* & $\begin{array}{l}\text { Adenocarcinoma } \\
\text { (grade group 2 or above) } \\
(\mathbf{n})\end{array}$ & $\mathbf{R R ( 9 5 \% ~ C I ) *}$ \\
\hline None $(<1 \%)$ & 10 & 1.00 & 3 & 1.00 \\
$1-5 \%$ & 62 & $1.89(0.87-4.11)^{*}$ & 37 & $3.70(1.08-12.66)^{*}$ \\
$>5 \%$ & 8 & $0.94(0.32-2.64)^{*}$ & 3 & $1.30(0.25-6.90)^{*}$ \\
All cases & 80 & - & 43 & - \\
\hline
\end{tabular}

* Compared with non-inflammation. RR, relative risk; CI, confidence interval. 

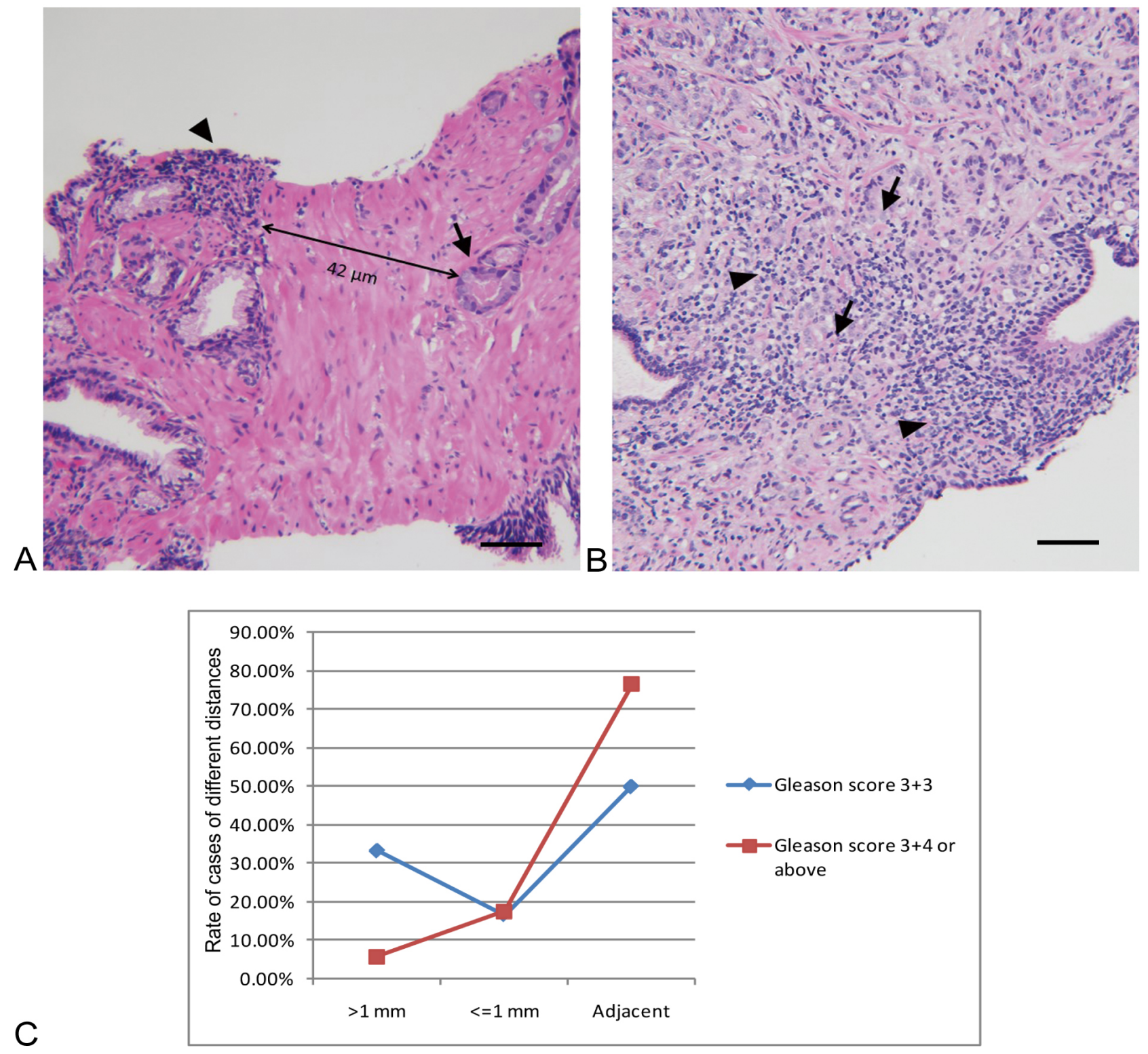

Figure 2. Needle biopsy specimen with inflammatory and adenocarcinoma cells. A. Representative H\&E-stained section of a needle biopsy of the prostate. Tumor cells (Gleason pattern $3+3$, indicated by arrowheads) are distinct from inflammatory cells (indicated by arrows). B. Tumor cells (Gleason pattern $4+5$, indicated by arrowheads) are mixed with inflammatory cells (indicated by arrows). H\&E stains, images were obtained at 100x magnification, scale bar $=20 \mathrm{um}$. C. Analysis of case rate of different distance between chronic inflammation and prostatic adenocarcinoma.

group 2 or above (RR 3.70, 95\% CI 1.08-12.66). After adjusting for age, ethnic group, PIN status and follow-up years, the overall RR of developing cancer for the inflammation group compared with the non-inflammation group was 1.52 (95\% CI 0.50-3.86), and the overall RR of developing cancer of grade group 2 or above for the inflammation group compared with the non-inflammation group was $2.76(95 \%$ CI $0.51-10.95)$

Distance between chronic inflammation and prostatic adenocarcinoma

In the 23 cases that exhibited cancer and chronic inflammation in the same prostate core, we analyzed the distance between the adenocarcinoma and inflammation (Table 5). Distances greater than $1 \mathrm{~mm}$ were observed in 3 cases, two of which were grade group 1. Distances less than or equal to $1 \mathrm{~mm}$ were observed in 4 cases, one of which was grade group 1 and 3 of which exhibited grade group 2 or above. Foci of inflammatory cells and tumor cells either admixed or immediately adjacent to one another were found in 16 of these 23 cases, and the majority (13/17, 76.5\%) exhibited adenocarcinoma of grade group 2 or above (Figure 2).

\section{Discussion}

Although the 5-year mortality rate of prostate cancer is not as high as other cancers, such as pancreatic adenocarcinoma, the number of afflicted men is quickly increasing due to the rapidly increasing population of males older than 50 years worldwide. Therefore, the identification of strategies for the prevention of prostate cancer remains an important medical challenge.

The potential relationship between chronic inflammation and prostatic adenocarcinoma has been a long-standing controversy. In 1994, a small nested case-control study within another Kaiser Permanente cohort study showed no association between 
Table 5. Distance between chronic inflammation and prostatic adenocarcinoma

\begin{tabular}{lllll}
\hline Distance & Case (n) & Gleason score 3 + 3 (n, \%) & Gleason score 3+ $\mathbf{4}$ or above (n, \%) & $P$ value \\
\hline Adjacent & 16 & $3(50.0)$ & $13(76.5)$ & 0.15 \\
$<=1 \mathrm{~mm}$ & 4 & $1(16.7)$ & $3(17.6)$ & - \\
$>1 \mathrm{~mm}$ & 3 & $2(33.3)$ & $1(5.9)$ & - \\
\hline
\end{tabular}

prostatitis and prostate cancer, as well as a non-significant positive association between gonorrhea and prostate cancer [11]. The Health Professional Follow-up Study reported no overall association between prostatitis, gonorrhea, syphilis, and prostate cancer [12]. However, in those studies, participants were asked to report their histories of gonorrhea, syphilis, and clinical prostatitis via a mailed questionnaire. The recall bias by patients impaired the study accuracy. With serological testing, the Prostate, Lung, Colorectal, and Ovarian (PLCO) Cancer Screening trial reported a borderline positive association in patients with any sexually transmitted diseases identified by serological evidence [13]. The presence and degree of chronic prostatic inflammation has been assessed histologically and reported in most recent studies, including in our current and previous cohorts [14-16]. A retrospective case control study performed by Gurel et al. revealed that chronic inflammation was common in benign prostate tissue and positively associated with prostate cancer [14]. In their study, chronic inflammation in prostate biopsy cores was scored by severity (grade 1-3) and was described in relation to the tissue compartment involving inflammation. Although they did not specify the percentage of tissue involving inflammation, their severity grading system was similar to ours. Gurel et al. noted that cancer with Gleason scores greater than 7 had a strong association with inflammation [14]. In a recent study of 285 cases, Platz et al. reported that the risk of developing prostate cancer appeared to increase with an increasing mean percentage of tissue area with inflammation, reporting statistically significant findings only for Gleason score $<4+3$ disease (grade groups 1 and 2) [17].

Our data indicate that prostatic adenocarcinoma (grade group 2 or above) has an association with chronic inflammation. In comparison to patients with no inflammation in their initial biopsies, patients with inflammation are 3 times more likely to develop prostate adenocarcinoma of grade group 2 or above. This finding is consistent with other published studies $[14,15]$. Although a Gleason score of $3+3$ is included in the new prostatic cancer grading system as ISUP grade group 1, questions have arisen concerning whether it should be considered prostate cancer due to its favorable prognosis compared with grade group 2 or above [18]. A very low rate of grade progression from $3+3$ to higher grades has been reported [19]. It is unclear whether chronic inflammation has an association with the development of grade group 1 prostate cancer. Based on our findings, we hypothesize that chronic inflammation causes mutations and other forms of damage to prostate cells, stimulating them to undergo changes that promote their survival and proliferation, thereby increasing the risk of prostate cancer. Recently, an "injury and regeneration" model has been proposed by De Marzo, which suggests that repeated bouts of injury to the prostatic epithelium, either due to inflammation or autoimmune disease, results in the proliferation of epithelial cells and uncontrolled growth. Several inflammatory molecular pathways have been implicated in this process, including MIC1, interleukin 1 receptor antagonist (IL1RN) and members of the Toll-like receptor (TLR) family, which were identified in the Cancer Prostate Sweden Study, a large-volume case-control study of prostate cancer [20]. Under the influence of an inflammatory microenvironment, the molecular phenotype of injured epithelial cells becomes altered. For example, data from one of our recent related studies have shown an increase in the expression of Bcl-2, a marker of cell survival, in epithelial cells in areas adjacent to chronic inflammation [21]. We contend that as their molecular phenotypes change, these particular epithelial cells acquire neoplastic characteristics, changing into precursor cells of malignancy, which eventually proliferate uncontrollably and develop the ability to invade adjacent tissue.

It is estimated that approximately $20 \%$ of all human cancers are caused by chronic infection and/or inflammation [5]. In fact, extensive work has been performed to investigate a possible link between inflammation and prostate cancer. To the best of our knowledge, our study is the first to evaluate and report the distance between inflammatory foci and tumor cells in human prostate cancer specimens. In 17 cases of grade group 2 or above, we found foci of inflammatory cells and tumor cells either admixed or immediately adjacent to one another in the majority of specimens (13/17), and a distance $<1 \mathrm{~mm}$ between cancer and inflammatory cells in another 3 cases (3/17). In contrast, in cancers of grade group 1 , only $50 \%(3 / 6)$ showed inflammation encroaching on cancer, and in 2 of 6 cases, the cancer and inflammation were greater than $2 \mathrm{~mm}$ apart. Our findings suggest that chronic inflammation, in the form of infiltrates of lymphocytes and macrophages, plays a direct role in the carcinogenesis of invasive prostate cancer. The normal prostate has been shown to contain endogenous inflammatory cells consisting of scattered stromal and intraepithelial lymphocytes and macrophages, which can increase in number in response to various stimuli. Analysis of the lymphocyte populations present in prostates affected by benign prostatic hyperplasia (BPH) has indicated that $70-80 \%$ of these cells are T cells and $10-15 \%$ are B cells. The T cells present in hyperplastic prostate tissue exhibit an inverted CD4:CD8 ratio: the majority are CD4-positive [22-24]. It is unclear whether the inflammatory infiltrates so commonly identified in prostates affected by BPH are triggered by foreign antigens, autoantigens, or both. Studies of the immunophenotypic and biological characteristics of the inflammatory cell infiltrates that accompany prostate cancer are limited, and thus further research is warranted.

Crowell et al. reported that progenitor-like luminal cells, marked by low expression of CD38 and spatially located near sites of inflammation, can initiate human prostate cancers exhibiting aggressive biological behavior [25]. Although it is unknown whether other types of inflammatory cells play a similar role in carcinogenesis, mast cells have been shown to stimulate cancer development in several in vivo models, probably as a result of the release of factors such as tumor necrosis factor-alpha and various proteases [26]. Another study demonstrated significantly higher prevalence of CD4+CD25 high T cells in cancerous compared withbenign tissues from the same prostate, as well as increased numbers in the peripheral blood of these cancer patients [27]. Our group has identified a significant accumulation of intraluminal and peri-glandular IL-17-producing cells in the mononuclear cell infiltrates associated with PIA lesions. Glandular and peri-glandular CD68+ macrophages and neutrophils are 
the predominant IL-17-producing cells in PIA lesions [28]. The accumulation of IL-17-expressing cells in PIA lesions provides direct evidence of an inflammatory microenvironment that may support the development of prostate cancer. Further studies of the possible role of $\mathrm{T}$ cells and other immune cells in prostate carcinogenesis appear to be warranted.

We have previously reported a higher incidence of subsequently diagnosed prostate cancer in patients whose prostate biopsies were initially benign but showed chronic inflammation, in comparison to patients whose prostate biopsies were initially benign but showed little or no inflammation, in a 5-year follow-up study of needle biopsy specimens from 177 patients who underwent prostate biopsy to investigate clinical concerns for malignancy [16]. In the current study, we increased the number of patients in our follow-up cohort and investigated the possible influence of inflammation on cancers of different Gleason grades. HGPIN is considered a putative lesion of prostate cancer that increases the risk for prostate adenocarcinoma, and thus our premise is that it is appropriate and acceptable to observe cases with HGPIN in the benign category. Per Dr. Jonathan Epstein in his assessment of HGPIN, the median risk recorded in the literature for cancer following the diagnosis of HGPIN upon needle biopsy is $24.1 \%$, which is not much higher than the risk reported in the literature for repeat biopsy following a benign diagnosis [9]. Furthermore, we evaluated the distances between inflammatory cell infiltrates and aggregates of cancer cells in the biopsy cores; to our knowledge, this type of analysis has not been previously reported.

Our study has some limitations. Although our statistical analyses indicate that patients with chronic prostatic inflammation are more likely to develop prostate cancer, and cancers with a higher Gleason grade, than a similar group of patients without chronic prostatic inflammation, the statistical validity of our data becomes less compelling after adjustment for age, ethnic group, PIN statusand number of years of follow-up. This is a common limitation in relatively small cohort studies. Adjustment for possible confounding factors using larger sample sizes may substantiate our findings. For future studies, we plan to continue accumulating data by increasing the number of followed patients.

Based on our current data and De Marzo's proposed injury and regeneration model, we hypothesize that an inflammatory microenvironment characterized by the accumulation of various types of immune cells is rich in reactive oxygen and nitrogen species, inducing oxidative stress in the proliferating epithelium that may interact with DNA to generate permanent genomic alterations. It would be of great interest to perform phenotypic analyses of the malignant cells in our biopsy cases, especially those that are intimately admixed with adjacent inflammatory cell infiltrates. Analyses of IC1, ILIRN and members of the Toll-like receptor family together with inflammatory signaling pathways is a logical next step. However, inflammation is an extremely complex process that involves various signaling pathways and several mediators. We speculate that numerous genes in the inflammatory pathways contribute to the pathogenesis of prostate cancer, and our future studies will focus on this area of research.

\section{Conclusions}

The present study identifies chronic inflammation as a contributor to prostate carcinogenesis. The presence and extent of chronic inflammation might be considered new criteria in the development and progression of prostate cancer.

\section{Acknowledgements}

Data included in this manuscript was presented by Liwei Jia, MD, $\mathrm{PhD}$ at the American Urology Association 2017 Annual Meeting in Boston, MA.

\section{Funding}

This research did not receive any specific financial support from funding agencies in the public, commercial, or not-for-profit sectors. Co-author SG acknowledges partial support of efforts by Department of Defense grant W81XWH-15-1-0558.

\section{Ethics approval and consent to participate}

This study was approved by the Institutional Review Board of University Hospitals(IRB number 02-14-36).

Each patient was informed of the study and signed an informed consent form and had the right to withdraw from the study at any time.

\section{Contributions of each author}

LJ: Case selection and accrual. LJ, WC and GTM: Morphologic evaluation of case slides. WC: Data analysis and manuscript writing. SG and GTM: Research design, manuscript revision and whole process supervision.

\section{Competing interests}

The authors declare no conflict of interest with the work.

\section{References}

1. Siegel RL, Miller KD, Jemal A: Cancer Statistics, 2017. CA Cancer J Clin 2017, 67(1): 7-30

2. Wang K, Karin M: Tumor-Elicited Inflammation and Colorectal Cancer. Adv Cancer Res 2015, 128: 173-196.

3. Berasain C, Castillo J, Perugorria MJ, Latasa MU, Prieto J, Avila MA: Inflammation and liver cancer: new molecular links. Ann N Y Acad Sci 2009, 1155: 206-221.

4. Coussens LM, Werb Z: Inflammation and cancer. Nature 2002, 420(6917): 860-867.

5. De Marzo AM, Platz EA, Sutcliffe S, Xu J, Grönberg H, Drake CG, Nakai Y, Isaacs WB, Nelson WG: Inflammation in prostate carcinogenesis. Nat Rev Cancer 2007, 7(4): 256-269.

6. Gonzalgo ML, Isaacs WB: Molecular pathways to prostate cancer. J Urol 2003, 170(6 Pt 1): 2444-2452.

7. Putzi MJ, De Marzo AM: Morphologic transitions between proliferative inflammatory atrophy and high-grade prostatic intraepithelial neoplasia. Urology 2000, 56(5): 828-832.

8. Young RH, Srigley JR, Amin MB Ulbright TM and Cubilla AL: Atlas of Tumor Pathology: Tumors of the Prostate Gland, Seminal Vesicles, Male Urethra and Penis,3rd Series. Washington, D. C.: Armed Forces Institute of Pathology, 1998.

9. Epstein JI, Herawi M: Prostate needle biopsies containing prostatic intraepithelial neoplasia or atypical foci suspicious for carcinoma: implications for patient care. J Urol 2006, 175(3 Pt 1): 820-834 .

10. Epstein Epstein JI, Zelefsky MJ, Sjoberg DD, Nelson JB, Egevad L, Magi-Galluzzi C, Vickers AJ, Parwani AV, Reuter VE, Fine SW, Eastham JA, Wiklund P, Han M, Reddy CA, Ciezki JP, Nyberg T, Klein EA. A Contemporary Prostate Cancer Grading System: A Validated Alternative to the Gleason Score. Eur Urol 2016, 69(3): 428-435.

11. Hiatt RA, Armstrong MA, Klatsky AL, Sidney S: Alcohol consumption, smoking, and other risk factors and prostate cancer in a large health plan cohort in California (United States). Cancer Causes Control 1994, 5(1): 66-72.

12. Sutcliffe S, Giovannucci E, De Marzo AM, Leitzmann MF, Willett 
WC, Platz EA: Gonorrhea, syphilis, clinical prostatitis, and the risk of prostate cancer. Cancer Epidemiol Biomarkers Prev 2006, 15(11): 2160-2166.

13. Huang WY, Hayes R, Pfeiffer R, Viscidi RP, Lee FK, Wang YF, Reding D, WhitbyD, Papp JR, Rabkin CS: Sexually transmissible infections and prostate cancerrisk. Cancer Epidemiol Biomarkers Prev 2008, 17(9): 2374-2381.

14. Gurel B, Lucia MS, Thompson IM Jr, Goodman PJ, Tangen CM, Kristal AR, Parnes HL, Hoque A, Lippman SM, Sutcliffe S, Peskoe SB, Drake CG, Nelson WG, De Marzo AM, Platz EA: Chronic inflammation in benign prostate tissue is associated with high-grade prostate cancer in the placebo arm of the prostate cancer prevention trial. Cancer Epidemiol Biomarkers Prev 2014, 23(5): 847-856.

15. Cheng I, Witte JS, Jacobsen SJ, Haque R, Quinn VP, Quesenberry CP, Caan BJ, Van Den Eeden SK: Prostatitis, sexually transmitted diseases, and prostate cancer: the California Men's Health Study. PLoS One 2010, 5(1): e8736.

16. MacLennan GT, Eisenberg R, Fleshman RL, Taylor JM, Fu P, Resnick MI, Gupta S: The influence of chronic inflammation in prostatic carcinogenesis: a 5-year followup study. J Urol 2006, 176(3): 1012-1016.

17. Platz EA, Kulac I, Barber JR, Drake CG, Joshu CE, Nelson WG, Lucia MS, Klein EA, Lippman SM, Parnes HL, Thompson IM, Goodman PJ, Tangen CM, De Marzo AM: A Prospective Study of Chronic Inflammation in Benign Prostate Tissue and Risk of Prostate Cancer: Linked PCPT and SELECT Cohorts. Cancer Epidemiol Biomarkers Prev 2017, 26(10): 1549-1557.

18. Carter HB, Partin AW, Walsh PC, Trock BJ, Veltri RW, Nelson WG, Coffey DS, Singer EA, Epstein JI: Gleason score 6 adenocarcinoma: should it be labeled as cancer? J Clin Oncol 2012, 30(35): 4294-4296.

19. Sheridan TB, Carter HB, Wang W, Landis PB, Epstein JI: Change in prostate cancer grade over time in men followed expectantly for stage T1c disease. J Urol 2008, 179(3): 901-904.

20. Zheng SL, Augustsson-Bälter K, Chang B, Hedelin M, Li L, Adami HO, Bensen J, Li G, Johnasson JE, Turner AR, Adams TS, Meyers DA, Isaacs WB, Xu J, Grönberg H: Sequence variants of toll-like receptor 4 are associated with prostate cancer risk: results from the Cancer Prostate in Sweden Study. Cancer Res 2004, 64(8): 29182922.

21. Glover M, Soni S, Ren Q, Maclennan GT, Fu P, Gupta S: Influence of chronicinflammation on Bcl-2 and PCNA expression in prostate needle biopsy specimens. Oncol Lett 2017, 14(4): 3927-3934.

22. Steiner GE, Djavan B, Kramer G, Handisurya A, Newman M, Lee $\mathrm{C}$, Marberger M: The picture of the prostatic lymphokine network is becoming increasingly complex. Rev Urol 2002, 4(4): 171-177.

23. Steiner GE, Newman ME, Paikl D, Stix U, Memaran-Dagda N, Lee $\mathrm{C}$, Marberger MJ: Expression and function of pro-inflammatory interleukin IL-17 and IL-17 receptor in normal, benign hyperplastic, and malignant prostate. Prostate 2003, 56(3): 171-182.

24. Steiner GE, Stix U, Handisurya A, Willheim M, Haitel A, Reithmayr F, Paikl D, Ecker RC, Hrachowitz K, Kramer G, Lee C, Marberger M: Cytokine expression pattern in benign prostatic hyperplasia infiltrating T cells and impact of lymphocytic infiltration on cytokine mRNA profile in prostatic tissue. Lab Invest 2003, 83(8): 1131-1146.

25. Crowell PD, Goldstein AS: Functional evidence that progenitor cells near sites of inflammation are precursors for aggressive prostate cancer. Mol Cell Oncol 2017, 4(2): e1279723.

26. Malaviya R, Ikeda T, Ross E, Abraham SN: Mast cell modulation of neutrophil influx and bacterial clearance at sites of infection through TNF-alpha. Nature 1996, 381(6577): 77-80.

27. Miller AM, Lundberg K, Ozenci V, Banham AH, Hellström M, Egevad L, Pisa P: CD4+ CD25 high T cells are enriched in the tumor and peripheral blood of prostate cancer patients. J Immunol 2006, 177(10): 7398-7405.

28. Vykhovanets EV, Maclennan GT, Vykhovanets OV, Gupta S: IL-
17 Expression by macrophages is associated with proliferative inflammatory atrophy lesions in prostate cancer patients. Int J Clin Exp Pathol 2011, 4(6): 552-565. 